El ectron- Hol e Di srupti on due to I on Mti on and For mat $i$ on of Coupl ed El ect r on $\mathrm{Hol}$ e and I on- Acoust i c Sol i ton i n a Pl asma

\begin{tabular}{|l|l|}
\hline 著者 & Saeki Koi chi , Genma Hi toshi \\
\hline $\begin{array}{l}\text { j ournal or } \\
\text { publ i cat i on ti tl e }\end{array}$ & Physi cal Revi ew Let t er s \\
\hline vol une & 80 \\
\hline nunber & 6 \\
\hline page r ange & 1224 1227 \\
\hline year & 1998 02 \\
\hline 出版者 & Aner i can Physi cal Soci et y \\
\hline 権利 & (c) 1998 The Aner i can Physi cal Soci et y \\
\hline URL & ht t p: //hdl . handl e. net /10297/609 \\
\hline
\end{tabular}




\title{
Electron-Hole Disruption due to Ion Motion and Formation of Coupled Electron Hole and Ion-Acoustic Soliton in a Plasma
}

\author{
Koichi Saeki and Hitoshi Genma* \\ Department of Physics, Faculty of Science, Shizuoka University, Ohya 836, Shizuoka 422-8017, Japan
}

(Received 30 September 1997)

\begin{abstract}
A computer simulation reveals that an electron phase-space hole whose velocity is of the order of or slower than the ion-acoustic speed strongly interacts with ions in a collisionless plasma. The ion motion leads to the disruption of an electron hole into two holes, and forms new coupled states of electron holes and ion-acoustic solitons. This dynamics can be explained by a theory using Sagdeev's potential. In the case of a bigger hole, it is entirely broken into many small holes. [S0031-9007(98)05326-5]

PACS numbers: 52.35.Sb, 52.35.Ra, 52.65.Rr
\end{abstract}

The hole (phase-space hole, phase-space vortex) is one of the most important Berstein-Greene-Kruskal solutions in a Vlasov plasma as well as the double layer [1]. Holes were found in computer runs simulating two stream instability [2]. Experimentally, an electron hole was discovered in a collisionless plasma of the $Q$ machine [3]. An asymmetric electron hole was also detected at the nonlinear stage of Buneman instability [4]. The ion version of the hole was observed in the double-plasma machine [5]. This ion hole has an important role on the anomalous electrical resistivity and the formation of ion-acoustic double layers [6] and double layers [7]. On the other hand, the data of the spacecraft GEOTAIL recently revealed that most of the broad-band electrostatic noise in the plasma of the geomagnetic tail is a series of electron holes [8].

Under the assumption of immovable ions, Schamel clarified that the propagation velocities of electron holes are limited below 1.3 times the electron thermal velocity [1]. Thus, the electron hole whose velocity is much smaller than the electron thermal speed or zero can exist in the time scale of electron motion. During the succeeding time scale of ion motion, we may imagine the strong interaction between the electron hole and its surrounding ions. Recently computer simulations elucidated the electron-hole dynamics including ion motion. An electron hole of a slow velocity induces the emission of an ion-acoustic-wave wake, and is accelerated due to the energy loss to excite the wake [9]. Electron holes excited by the electron two-stream instability are filled up with electrons in phase space and are damped because of ionacoustic-wave emission [10].

In this Letter, we present the first observation of the electron-hole disruption and the formation of new coupled states of electron holes and ion-acoustic solitons (abbreviated to coupled hole-solitons or CHSs) in computer runs with movable ions. These CHSs are well explained by the theory of Saeki and Rasmussen [11].

We adopt the electrostatic simulation of the particle-incell model with one dimensional coordinate $x$. In the case of immovable ions (mass ratio of ion to electron $m_{i} / m_{e}=$ $\infty$ ), the temporal behavior of an excited initial electron hole with a propagation velocity $u_{\text {ini }}=0$ is shown in phase space $\left(x / \lambda_{D}, v_{e} / v_{t e}\right)$ of Fig. 1(a). Here, $v_{e}, v_{t e}=$ $\sqrt{k T_{e} / m_{e}}$, and $\lambda_{D}=v_{t e} / \omega_{p e}$ are electron velocity, electron thermal velocity, and electron Debye length, respectively. $k$ is Boltzmann's constant. $T_{e}$ is electron temperature. $T=\omega_{p e} t / 2 \pi$ is normalized time. $\omega_{p e}$ and $t$ are plasma angular frequency and time, respectively. The system length is $125 \lambda_{D}$ and consists of 3000 cells. Each number of ions and electrons per unit cell is 1000 . The program runs with the time step $\Delta T=0.25 / 2 \pi$.

In order to create a self-consistent hole and prevent the simultaneous wave excitation, we construct the electron hole at $T=0$ in Fig. 1(a) by enlarging the hole in phase space artificially in the time scale much slower than $2 \pi / \omega_{p e}$ while $T<0$. This quasistatic hole formation is useful as shown in Fig. 1(a). Because of no interaction with ions, the hole is almost in a steady state except small oscillations of aspect ratio and axis angle of the elliptic hole in phase space. Here, the plasma potential $\phi$ is not shown, but is the same to the potential at $T=0$ of Fig. 1(c), where the half width of the potential $\Delta x_{\text {ini }}$ is $5 \lambda_{D}$, and the maximum normalized potential $\Phi_{\text {ini }}$ $\left(=e \phi_{\max , \text { ini }} / k T_{e}\right)$ is 1.2 for the initial electron hole.

On the other hand, the stability of this electron hole in the case of movable ions $\left(m_{i} / m_{e}=100\right)$ is demonstrated in Figs. 1(b) and 1(c). The temperature ratio of electron to ion $T_{e} / T_{i}$ is 40 . The other simulated conditions are just the same as the case of Fig. 1(a) except that ions start to be movable at $T=0$. The spatial structures of the normalized plasma potential $e \phi / k T_{e}$, the electron phase fluid in phase space $\left(x / \lambda_{D}, v_{e} / v_{t e}\right)$, and the ion phase fluid in phase space $\left(x / \lambda_{D}, v_{i} / c_{s}\right)$ are shown in Fig. 1(c). Here, $v_{i}$ is the ion velocity, and $c_{s}=\sqrt{k T_{e} / m_{i}}$ is the ion acoustic speed. The positive potential of the electron hole causes the compression of ions on both sides of the hole. The resultant ion perturbations deform the hole itself and disrupt the hole into two holes. Apparently these two holes propagating in opposite directions to each other are accompanied by compressional ion pulses and form new solitary structures propagating stably. Each structure comprises an electron hole and an ion-acoustic soliton, 
a)

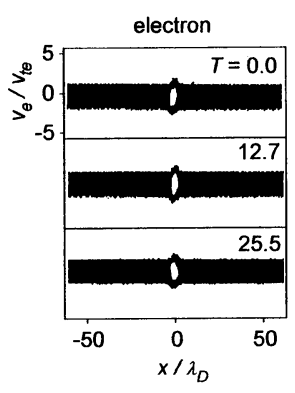

c)

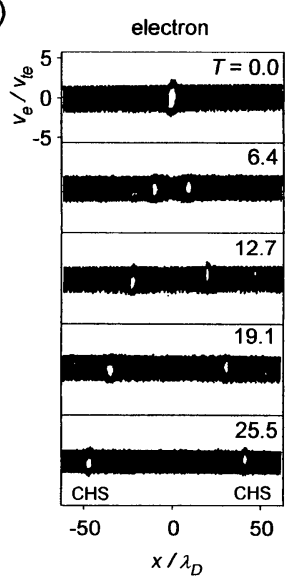

b)

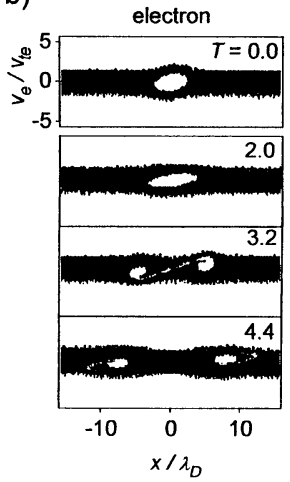

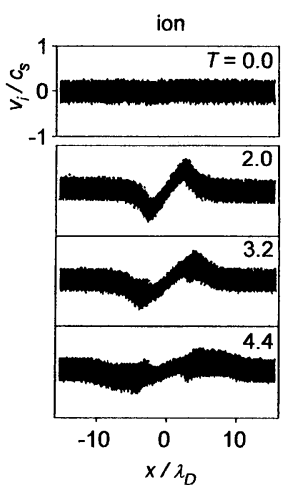

potential
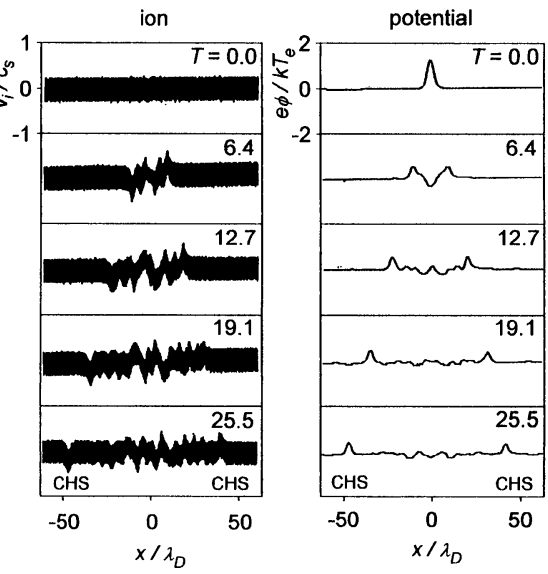

FIG. 1. Temporal behavior of an electron hole (a) with immovable ions, and (b), (c) with movable ions $\left(m_{i} / m_{e}=100\right)$. Maximum potential $\Phi_{\text {ini }}=1.2$, potential half width $\Delta x_{\mathrm{ini}}=5 \lambda_{D}$, and velocity $u_{\mathrm{ini}}=0$ for the initial hole. $T=\omega_{p e} t / 2 \pi$ is the normalized time. (b) Detail of hole disruption at the beginnings of (c). (c) Electron-hole disruption and formation of coupled hole-solitons observed in phase space $\left(x / \lambda_{D}, v_{e} / v_{t e}\right)$, in phase space $\left(x / \lambda_{D}, v_{i} / C_{s}\right)$, and as a spatial potential variation $e \phi / k T_{e}$. $M=3.0, \Phi_{\max }=0.50$, and $S=2.4$ for the left CHS. $M=2.8, \Phi_{\max }=0.45$, and $S=2.2$ for the right CHS.

being regarded as a coupled state of an electron hole and an ion-acoustic soliton, a coupled hole-soliton.

The early stage of this dynamics is shown in Fig. 1(b). Each compressional ion pulse induced on both sides by the positive potential of the initial electron hole has also a positive potential. This positive potential of each ion pulse pulls the electrons surrounding the hole from both sides of it. In other words, the electron hole behaves as a macroparticle having positive charge and negative mass [2], thereby being dragged by the positive potential of each ion pulse. As a result, the hole is elongated and divided into two holes. This drag is also the mechanism to maintain the stability of the CHS, where the positive potential of the ion-acoustic soliton attaches the electron hole to the soliton.

As shown in Fig. 1(c), the initial electron hole induces not only two CHSs but also ion-acoustic-wave wakes as already reported in [9]. The CHSs on the left-hand side and the right-hand side have Mach numbers $M\left(=u / c_{s}\right)$ of 3.0 and 2.8, respectively. Here, $u$ is the propagation speed of each CHS. This asymmetry of $M$ arises from the small oscillations of the initial hole mentioned before.

The dynamics of an initial electron hole propagating with $u_{\text {ini }} \neq 0$ is also investigated. If we vary only $u_{\text {ini }}$
$(>0)$ of the initial hole, the induced CHS on the righthand side $(x>0, u>0)$ has a bigger potential and propagates faster than that in the case of $u_{\text {ini }}=0$. The potential and the velocity of another CHS on the opposite side $(x<0, u<0)$ become smaller and slower, respectively. When we increase only $u_{\text {ini }}$ of the initial hole in Fig. 1(c) to $0.9 c_{s}$, the right $\mathrm{CHS}$ gets a Mach number of 3.9, and the left CHS almost disappears. The initial hole of $u_{\text {ini }}>c_{s}$ gives only the right CHS. According to the change of the initial holes in shapes (aspect ratios and areas in phase space) and in propagating velocities $u_{\text {ini }}$, we are able to observe various kinds of CHSs after their interaction with ions.

The experimental data of Fig. 2(b) obtained by these computer runs express the relation between the Mach number $M$ and the maximum normalized potential $\Phi_{\max }$ $\left(=e \phi_{\max } / k T_{e}\right)$ of CHSs with the area $S$ of the hole region in phase space as a parameter. Here, $S$ is normalized by $\lambda_{D} v_{t e}$. In order to compare the results with the theory described later, we put the electron velocity distribution function of the excited initial hole to be zero in the hole region. Thus, the resultant hole of each CHS has a sharp boundary surrounding the hole region in phase space. The data are plotted after selecting the CHSs having the same 
a)

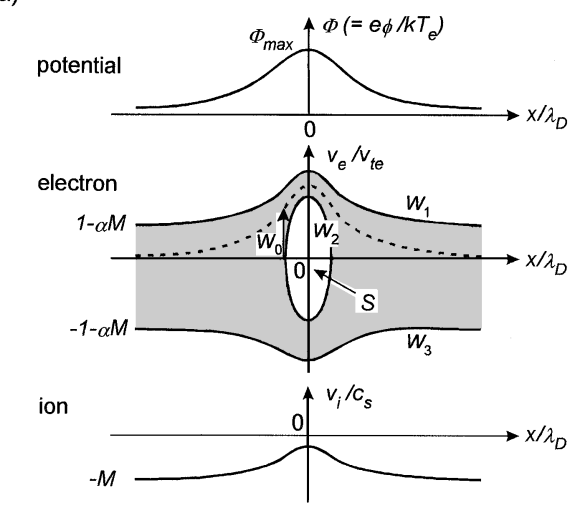

b)

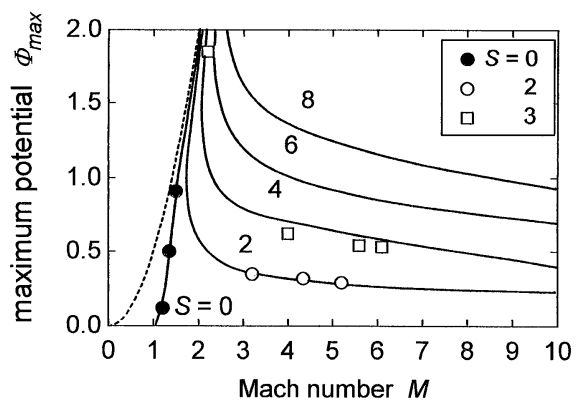

FIG. 2. (a) Spatial structure of a coupled hole-soliton (a coupled state of an electron hole and an ion-acoustic soliton) with a propagation speed $u$ under the water bag model. Upper, middle, and lower figures indicate potential, electron phase fluid, and ion phase fluid, respectively. $M=u / c_{s}, \Phi_{\max }=$ $e \phi_{\max } / k T_{e}, \quad \alpha=\left(m_{e} / m_{i}\right)^{1 / 2}, \quad$ and $\quad W_{0,1,2,3}=v_{0,1,2,3} / v_{t e}$. (b) Relation between the Mach number $M$ and the maximum potential $\Phi_{\max }$ of CHSs with the hole area $S$ in electron phase fluid as a parameter. $\bullet$, $\circ$, and $\square$ are experimental points. $m_{i} / m_{e}=100$. Here, CHSs of $S=0$ mean pure ion-acoustic solitons, and the dotted line $\left(\Phi=M^{2} / 2\right)$ indicates the critical line of ion reflection due to the CHS potential $\phi$.
$S$ of 2 and 3 accurately within $10 \%$, among the above various kinds of CHSs. The CHSs of $S=0$ mean pure ion-acoustic solitons with no electron hole being excited by applying an external positive potential pulse.

Now, we try to figure out the theoretical relation between the Mach number $M$ and the maximum potential $\Phi_{\max }$ of CHSs according to Ref. [11]. The spatial structure of a CHS in the moving frame of a CHS propagation velocity $u$ is illustrated in Fig. 2(a).

For simplicity, we assume a rectangular velocity distribution function of electrons; its spread and height are $2 v_{t e}$ and $1 / 2 v_{t e}$ at $x= \pm \infty$, respectively. Thus, we are able to calculate the spatial electron density variation $n_{e}(x)$ as a function of $\phi(x)$ from the electron velocities on the boundaries of the electron phase fluid $v_{1}, v_{2}$, and $v_{3}$, being the velocities on the upper, hole, and lower boundaries of the electron phase fluid, respectively; i.e., $\quad n_{e}(x)=n_{0}\left[v_{1}+\left|v_{3}\right|-2\left|v_{2}\right| \theta\left(v_{2}^{2}\right)\right] / 2 v_{t e}$. Here, $n_{0}$ is the plasma density at $x= \pm \infty$, and $\theta(x)$ is the step function. $v_{1}$ is derived as a function of $\phi(x)$ from the energy equation $(1 / 2) m_{e}\left(v_{t e}-u\right)^{2}=$ $(1 / 2) m_{e} v_{1}^{2}-e \phi(x) . \quad v_{2}$ and $\boldsymbol{v}_{3}$ are also obtained from $-(1 / 2) m_{e} v_{0}^{2}=(1 / 2) m_{e} v_{2}^{2}-e \phi(x)$ and from $(1 / 2) m_{e}\left(-\boldsymbol{v}_{t e}-u\right)^{2}=(1 / 2) m_{e} \boldsymbol{v}_{3}^{2}-\boldsymbol{e} \boldsymbol{\phi}(x), \quad$ respectively. $\quad v_{0}$ is the velocity $v_{e}$ of the electrons at $v_{2}=0$ that have no kinetic energy $\left(v_{e}=0\right)$ at $x= \pm \infty$.

The ion temperature is assumed to be zero. Therefore, the spatial dependence of ion density $n_{i}(x)$ as a function of $\phi(x)$ is also calculated from the energy equation, $(1 / 2) m_{i}(-u)^{2}=(1 / 2) m_{i} v_{i}^{2}-e \Phi(x)$, and the equation of continuity, $n_{0}(-u)=n_{i}(x) v_{i}$.

Poisson's equation, $\partial^{2} \phi(x) / \partial x^{2}=e\left[n_{e}(x)-n_{i}(x)\right] /$ $\epsilon_{0}$, relates $\phi(x)$ to $n_{i}(x)$ and $n_{e}(x)$. Normalizing the quantities as $z=x / \lambda_{D}, \Phi=e \phi / k T_{e}$, and $W_{0}=v_{0} / v_{t e}$, we obtain the following equation:

$$
\begin{aligned}
\frac{\partial^{2} \Phi}{\partial z^{2}}= & \frac{1}{2}\left\{\left[(1-\alpha M)^{2}+2 \Phi\right]^{1 / 2}+\left[(1+\alpha M)^{2}+2 \Phi\right]^{1 / 2}\right\}-\theta\left(-W_{0}^{2}+2 \Phi\right)\left[-W_{0}^{2}+2 \Phi\right]^{1 / 2} \\
& -M\left(M^{2}-2 \Phi\right)^{-1 / 2} .
\end{aligned}
$$

Here, $\alpha=\left(m_{e} / m_{i}\right)^{1 / 2}$. By integrating Eq. (1) multiplied by $\partial \Phi / \partial z$ from $-\infty$ to $z$, we derive Sagdeev's potential $V\left(\Phi, M, W_{0}, \alpha\right)[12]$

$$
\begin{aligned}
V\left(\Phi, M, W_{0}, \alpha\right)= & -\frac{1}{6}\left\{\left[(1-\alpha M)^{2}+2 \Phi\right]^{3 / 2}+\left[(1+\alpha M)^{2}+2 \Phi\right]^{3 / 2}-(1-\alpha M)^{3}-(1+\alpha M)^{3}\right\} \\
& +\frac{1}{3} \theta\left(-W_{0}^{2}+2 \Phi\right)\left[-W_{0}^{2}+2 \Phi\right]^{3 / 2}+M\left[M-\left(M^{2}-2 \Phi\right)^{1 / 2}\right] .
\end{aligned}
$$

Here, Eq. (2) is valid when $\alpha M<1$, and we put $V(0, M$, $\left.W_{0}, \alpha\right)=0 . V\left(\Phi, M, W_{0}, \alpha\right)$ satisfies $(1 / 2)(\partial \Phi / \partial z)^{2}+$ $V\left(\Phi, M, W_{0}, \alpha\right)=0$. The CHS has a maximum potential $\Phi_{\max }$ satisfying $V\left(\Phi_{\max }, M, W_{0}, \alpha\right)=0$.

On the other hand, the hole area $S$ of the CHS in the electron phase fluid is calculated as follows:

$$
S=4 \int_{W_{0}^{2} / 2}^{\Phi_{\max }}\left(\frac{-W_{0}^{2}+2 \Phi}{-2 V\left(\Phi, M, W_{0}, \alpha\right)}\right)^{1 / 2} d \Phi .
$$

Here, $S$ is a function of $\Phi_{\max }, M, W_{0}$, and $\alpha$. Thus, by eliminating $W_{0}$, the equation $V\left(\Phi_{\max }, M, W_{0}, \alpha\right)=0$ and Eq. (3) yield the relation between $M$ and $\Phi_{\max }$ with $S$ and $\alpha$ as parameters.

The theoretical $M-\Phi_{\max }$ relation of CHSs in the case of $\alpha=0.1\left(m_{i} / m_{e}=100\right)$ is plotted with $S$ as a parameter on Fig. 2(b). The dotted line expresses $\Phi=$ $M^{2} / 2$, and means the critical line of ion reflection due to the positive potential of CHSs. When $S$ becomes finite, 

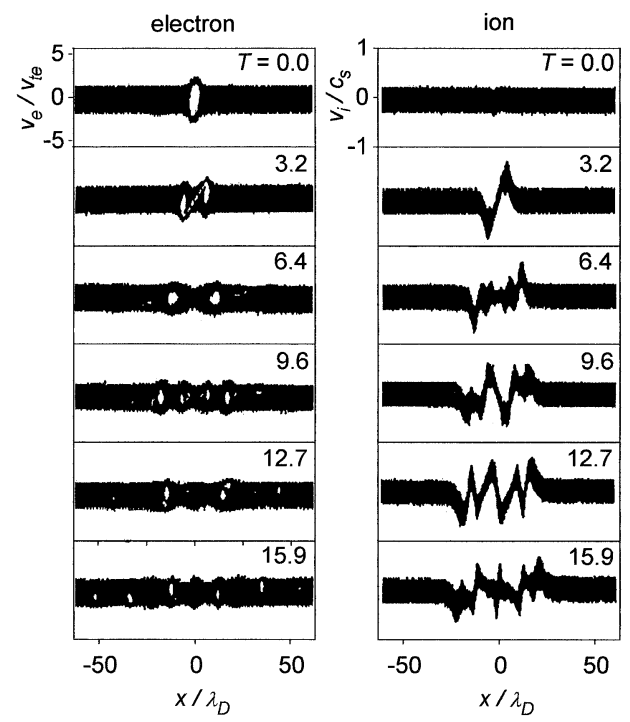

FIG. 3. Electron-hole disruption in the case of a big hole. $\Phi_{\text {ini }}=2.4, \Delta x_{\text {ini }}=5 \lambda_{D}, u_{\mathrm{ini}}=0$, and $m_{i} / m_{e}=100$.

the Mach number $M$ of the CHS increases. The aspect (height-to-width) ratio of the elliptic hole in the electron phase fluid grows along the theoretical curve of $S=$ const. with an increase in $\Phi_{\max }$. The large Mach number gives pure electron holes. Hence, Fig. 2(b) provides the unified description of pure ion-acoustic solitons $(S=0)$, coupled hole-solitons and pure electron holes $(M \gg 1)$, expanding from $M=1$ to $M=v_{t e} / c_{s}=\left(m_{i} / m_{e}\right)^{1 / 2}$. Thus, the larger mass ratio $m_{i} / m_{e}$ leads to the larger Mach number $M$ of the CHS. The theoretical curves well explain the experimental data.

In general, the wave in a collisionless plasma is the corrugation of the phase fluid, while the phase-space hole is the vortex of the phase fluid and does not belong to the wave category. The obtained CHS is a new coupled state of a wave (ion-acoustic wave) and a phase-space vortex (electron hole), propagating faster than the original wave speed $\left(=c_{s}\right)$. The similar physical concept is found in the drift-wave modon, which is a coupled state of a drift wave and a dipole vortex and propagates faster than the driftwave speed in a magnetized plasma [13]. Moreover, in contrast to the caviton induced by the interaction between an electron plasma wave and ions around it [14], this CHS is a result of the interaction of an electron hole with its surrounding ions.

When the initial electron hole has a bigger hole in phase space, the dynamics of the hole is very different from Fig. 1. Figure 3 is in the case of $\Phi_{\text {ini }}=2.4$. Each divided hole induces new ion compressional pulses and splits again into two holes. Thus, the hole is disrupted into many holes after the interaction with ions.

In conclusion, an electron hole whose velocity is of the order of or slower than the ion-acoustic speed strongly interacts with ions in a collisionless plasma and results in the hole disruption and the formation of new coupled states between electron holes and ion-acoustic solitons, CHSs. In the case of a bigger hole, it is entirely broken into many small holes.

One of the authors (K.S.) wishes to express his gratitude to Dr. V. A. Turikov for his simulation technique, and to Dr. S. Iizuka and Dr. J. J. Rasmussen for their discussions. He also thanks Dr. N. Sato, Dr. Y. Amagishi, and Dr. H. Nagashima for their encouragement.

* Present address: Data Communication System Co. Ltd., Shinbashi 5-23-4, Minatoku, Tokyo 105, Japan.

[1] H. Schamel, Phys. Rep. 140, 161 (1986).

[2] H.L. Berk and K. V. Roberts, Phys. Fluids 10, 1595 (1967); H. L. Berk, C. E. Nielsen, and K. V. Roberts, Phys. Fluids 13, 980 (1970).

[3] K. Saeki, P. Michelsen, H. L. Pecseli, and J. J. Rasmussen, Phys. Rev. Lett. 42, 501 (1979).

[4] Y. Takeda and K. Yamagiwa, Phys. Rev. Lett. 55, 711 (1985).

[5] H. L. Pécseli, R. J. Armstrong, and J. Trulsen, Phys. Lett. 81A, 386 (1981).

[6] T. Sato and H. Okuda, Phys. Rev. Lett. 44, 740 (1980).

[7] H. Fujita, S. Yagura, and K. Matsuo, Phys. Fluids 27, 2948 (1984).

[8] H. Matsumoto et al., Geophys. Res. Lett. 21, 2915 (1994). Their naming of electrostatic solitary waves gives confusion, because the hole is out of the wave category.

[9] J. M. Dawson, Phys. Plasmas 2, 2189 (1995).

[10] Y. Omura, H. Kojima, and H. Matsumoto, Geophys. Res. Lett. 21, 2923 (1994).

[11] K. Saeki and J. J. Rasmussen, J. Phys. Soc. Jpn. 60, 735 (1991).

[12] Review of Plasma Physics, edited by M. A. Leontovich (Consultants Bureau, New York, 1966), Vol. 4, p. 23.

[13] V.D. Larichev and G. M. Reznik, Dokl. Akad. Nauk SSSR 31, 1077 (1976).

[14] H. C. Kim, R. L. Stenzel, and A. Y. Wong, Phys. Rev. Lett. 33, 886 (1974). 